\title{
Architecture design of ad-hoc cognitive radio network
}

\author{
P. Surendar, A. Sridevi and R. Sona \\ Department of Electronics \& Communication Engineering \\ M. Kumarasamy College of Engineering (Autonomous), Karur - 639113, Tamil Nadu, India; \\ surendarp.ece@mkce.ac.in, sridevia.ece@mkce.ac.in, r.sonain@gmail.com
}

\begin{abstract}
Objectives: The target for effective spectrum utilization is termed as a essential factor in today's fast growing technology and its current vary distribution arrangements, the vary usage effectiveness at a particular time and space is low. The throughput and delay has to be analysed and thereby bandwidth should be properly utilized. Methods/Statistical Analysis: Cognitive Radio (CR) is estimated as the technology that will improve the making use of the spectrum resources in upcoming generation unwired systems. It'll sense a use spectrum in associate opportunist manner whereas not creating any damage to cognitive users. Prosper a cognitive feature radio approach strategy supported the exercise of a moving genetic formula for CR. Findings: The crossover operators as well as mutation operators are grown to remain the channel distribution set up constantly among the suitable state. Supported these methods, cheap employment is planned. Results display that our formula acquire acceptable results with all acceptable network interference and scale back the quantity of used channels. The cognitive radio is implemented using MATLAB and the energy efficiency analysis is done. The throughput and delay are analysed by simulation process using MATLAB. Application/Improvements: The aim of any routing protocol is to produce the fundamental Quality of Service (QoS) to the applications in terms of overall output and end-to-end delay. CRAHNs need capabilities to determine on the most effective spectrum band among the offered bands in line with the QoS necessities of the applications.
\end{abstract}

Keywords: Cognitive Radio, Throughput, Stackleberg Theory, CRAHN, GA, MATLAB

\section{Introduction}

Spectrum shortage is one in all the essential bottlenecks for the advancement of future remote correspondence frameworks. Below current vary distribution arrangements, the vary usage effectiveness in licensed vary at a particular time and space is low. The Federal Communications Commission (FCC) gauges the employment of relegated vary to be somewhere around V-day and eighty fifth to deal with such wastefulness given affected vary accessibility, the Federal Communications Commission has supported arrangements, as an example, deft utilization of licensed vary, as an example, TV white areas. Such arrangements are within the long haul acknowledged through the appropriation of psychological feature Radio (CR), innovation, that is purported to build vary use through component vary access (DSA) technique, whereby unaccredited (CR) purchasers sagaciously utilize licensed bands once not possessed. Hence, the usage of vary will be very upgraded by sharp correspondence on licensed vary.

Cognitive Radio Ad-Hoc Networks (CRAHNs) have pulled in much consideration in the exploration group lately. Dissimilar to either customary CR systems or impromptu systems, CRAHNs give a non-base backing and range heterogeneity based remote system which raises one of a kind issues and difficulties ${ }^{1-3}$. 
Two particular sorts of steering conventions have been examined: Helpful directing and non-agreeable directing conventions. An appropriated CR steering convention is to indicate the issues of PU collector insurance, administration separation in CR courses, and joint range course choice. A helpful steering Convention has been considered for accomplishing higher channel limit pick up. Because of range heterogeneity attributes, the channel which gives most extreme limit is chosen to transmission in each immediate connection, and the hub that can give the greatest limit increase is chosen as the hand-off hub for helpful steering.

In order to overcome the problem faced by the conventional Spectrum aggregated CRAHN method Stakleburg Gaming theory will be implemented in order to achieve high throughput and reduced delay this also improves the spectrum and energy efficiency. In this Gaming theory will be implemented and by this the game will be conducted between different channels. After conducting the game winner node among each channel are shortlisted. Now the transmission will be towards the Base station, yet again the gamming progression is done and among the shortlisted nodes the node with high strength is being denoted and this is considered as the path with high transmitting Energy and efficiency. By choosing this path we can able to increase the Efficiency of the system.
The Existing system is termed as Spectrum Aggregation standed Cooperative direction-finding Protocol for CRAHNs. The first commitments are given in 3 layers as shown in Figure 1. We tend to begin our exchange with the vary assortment system for CRAHNs. It incorporates the define of PHY and mac layer expected to total distinctive vary groups/channels. After this, we tend to propose 3 numerous vary conglomeration calculations. The first calculation decrease the communicate power for CR shoppers seeable of a rate request.

The second calculation amplifies the entire channel limit for a CR consumer. At long last, the third calculation minimizes the top-to-end idleness for the cr system. Supported the vary total calculations, we tend to set up a useful guiding convention, termed as spectrum aggregation standed cooperative direction-finding protocol.

In addition to the current system gamming theory has been enforced so as to reinforce the performance and spectral potency of the system. Stackleberg gamming theory is introduced so as to get the effective node with high strength and high information transferring capability ${ }^{4,5}$. During this cooperative routing protocol is enforced to get the shortest path. this technique primarily deals with the SU in numerous channels those that get access to the spectrum square measure evaluated among them and therefore the winner node is left to speak with the

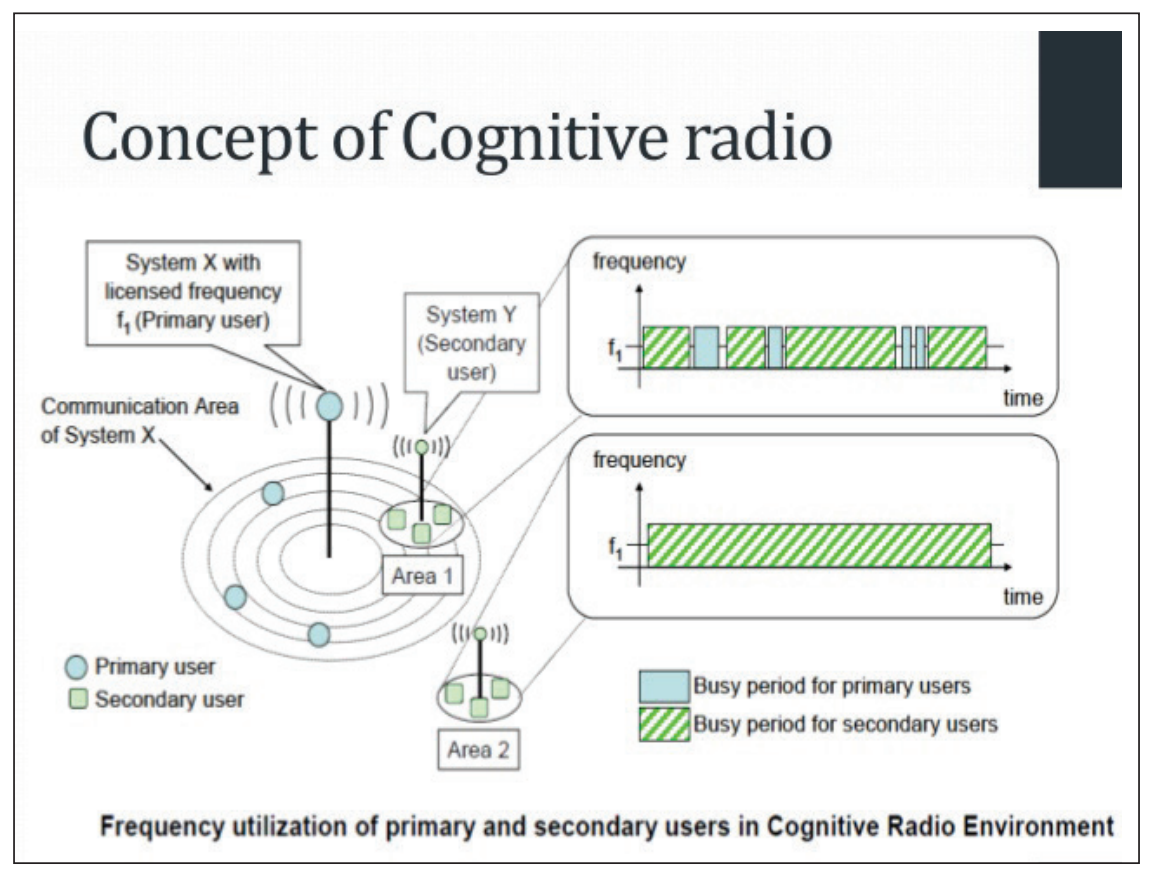

Figure 1. Methodology for CRN. 
destination this technique will overcome the drawbacks featured by the Priority based mostly system.

The over read of this paper is given as follows, Section 2 deals with the system analysis, Section 3 deals with the experimental result, Section 4 covers the conclusion half.

\section{System Analysis}

In changing channel allocation, offered spectrum is split into a bunch of spectrum bands that take issue from one another in information measure and transmission vary every subordinate user keeps an inventory of accessible channels. Subordinate users square measure assigned utterly different offered spectrum's supported their location, radio interface, and different needs, and can bear in mind of their position with relevancy the encircling basic users as they cant use a channel occupied by a basic user. Some variables square measure outlined as follows for system mode

1. $\mathrm{U}(0,1,2, \ldots, \mathrm{U}-1)$ be the number of subordinate users.

2. The edges be graphically by the $\mathrm{U} \times \mathrm{U}$ matrix $\mathrm{E}$, where $\mathrm{m}, \mathrm{n}=$ one if there is a grip amidst vertices $\mathrm{m}$ and $\mathrm{n}$, and $\mathrm{e}, \mathrm{m}, \mathrm{n}=$ zero implies that $\mathrm{m}$ and $\mathrm{n}$ use identical frequencies.

3. W $(0,1,2, \ldots, \mathrm{W}-1)$ be the number of unoccupied channels.

4. $\mathrm{Q}=\mathrm{dn} \in \mathrm{U}$ be printed as a result of the demand matrix of users, and dnact as the data rate of user $n$.

5. $\mathrm{R}=\ln , \mathrm{m} \in \mathrm{U} \times \mathrm{W}$ characterize the per user obtainable spectrum, i.e. spectrum band $\mathrm{m}$ is on the marketplace for user $\mathrm{u}$ if $\ln , \mathrm{m}=1$.

6. $\mathrm{T}=\mathrm{Cu}, \mathrm{k}, \mathrm{m} \in \mathrm{U} \times \mathrm{U} \times \mathrm{W}$ represent the interference constraint, where if $\mathrm{Cu}, \mathrm{k}, \mathrm{m}=1$, users $\mathrm{u}$ and $\mathrm{k}$ will cause interference as they used the spectrum band $\mathrm{m}$ at same time. In this the constraints square measure spectrum band specific. Note that 2 users World Health Organization square measure forced by a spectrum band is not been able to use this band at the identical time.

7. $\mathrm{B}=\mathrm{U} \times \mathrm{W}$ describe the reward that a user u gets by successfully effort offered spectrum band m, i.e. bu, mre presents the foremost bw/throughput that may be non ancestral (considering no interference from different neighbors).

8. Pound $=\mathrm{U} \times \mathrm{W}$ represent the out flip or the information measure each of each channel that's on the marketplace for every user to use. Performance of allocation could also be expressed as follows in keeping with the on prime of definitions: The complete metric of the system.

In addition to the current system gamming theory has been enforced so as to boost the performance and spectral potency of the system. Stackleberg gamming theory is introduced so as to get the effective node with high strength and high information transferring capability. During this cooperative routing protocol is enforced to get the shortest path. This method primarily deals with the SU in varied channels people who get access to the spectrum area unit evaluated among them and therefore the winner node is left to speak with the destination. This method will overcome the drawbacks Janus-faced by the Priority based mostly system $\frac{6,7}{2}$.

The over read of this paper is given as follows, Section 2 deals with the system analysis, Section 3 deals with the Experimental Result, Section 4 covers the conclusion half.

\section{Evaluate the Performance}

It is important to calculate the accomplishment of the projected GA, we have an inclination to tend to use laptop computer simulations, that we have an inclination to examine the proportion of detached interference by the rule once the quantity of nodes and collectively the offered channels will increase. This means of arriving was designed to degree impact on a frequency for every expedient user below the first users by employing a standardized value performs to pick out Associate in Nursing offered channel for every state of affairs, we have an inclination to tend to indiscriminately create a particular interference topology visual representation instances, with the quantity of nodes varied from ten to sixty, with the meddling ranges written throughout a really constraint matrix, where: every channel is engaged by a main user (Primary User) throughout a really positive vary. Thus every node inside a chemical element's vary can't utilize the channel engaged by this plutonium. Every node among the network has its particular offered channel set, per the positions of the PUs. Some nodes will use constant channel throughout a really positive vary. The collision of the moving occupation of frequency by the primary users was sculptured considering a mean inactive time a minimum of 10 times larger than the time required for 
the rule to succeed in the stopping criterion. The channel assignment flinch of this network is resolved by the projected rule, and analysed, for 3 altogether utterly totally different scenarios: one. Varied the quantity of accessible channels K; a pair of. Varied the quantity of network nodes $\mathrm{N}$ three. For every instances, we have an inclination to tend to remark that the quantity of nodes and collectively the offered channels force the potency of the ways in which and collectively the network interference. From the figure the accomplishment in terms of detached interference decreases once the quantity of nodes will increase, and every node has less potentialities to travel searching associated use Associate in Nursing tabu channel ${ }^{\circledR}$. Once the quantity of accessible channels will increase, every channel includes plenty of potentialities to be appointed to altogether utterly totally dissimilar channels for interference dodging. thus the impact caused by the variation among the vary of accessible channels once and earlier the channel allocation and collectively the appearance at the staying criterion agree to keeping stable throughout all simulated cases, the quantity of accessible channels varied from a pair of to ten $\frac{9,10}{}$.

\section{System Analysis}

\subsection{Stackelberg Gaming Theory}

The Stackelberg management representation (Figure 2) is a planned game in economics in which the skull firm moves main and then the follower firms move in sequence. In game theory terms, the troupe of this game is a leader and a follower and they compete on quantity. The Stackelberg leader is occasionally referred to as the market leader operation of game theory as shown in the figure.

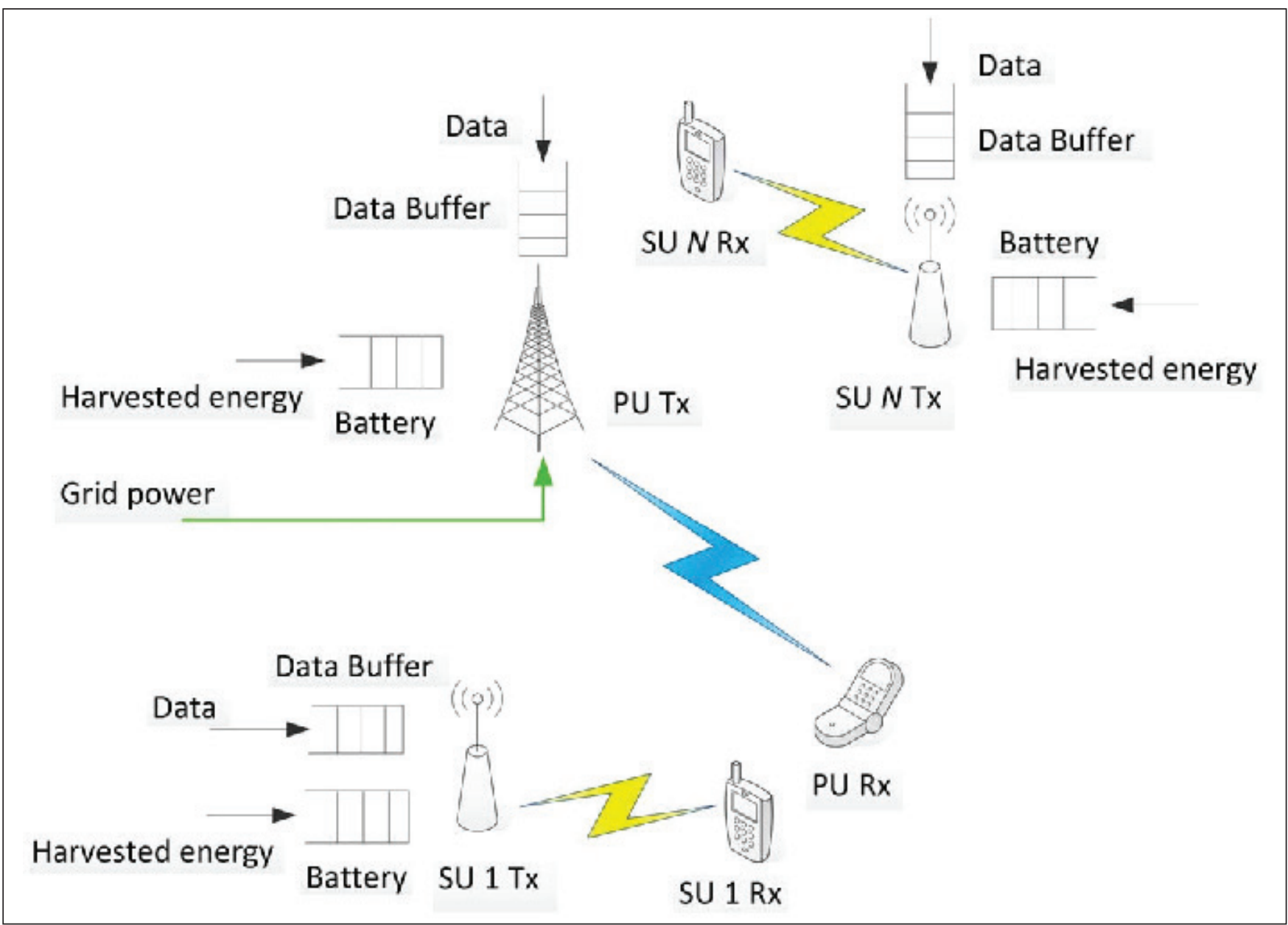

Figure 2. Operation of Stackelberg game theory. 


\section{Experimental Result}

In this section, the analysis is performed for the obtained experimental results. The Matlab GUI implementation of cognitive radio technology is given in Figure 3. The simulation result (Figure 4) shows the existing and proposed implementation of transmission power. The proposed method provides lesser power to transmute in CRN network. The result for energy efficient channel selection is analyzed a shown in Figure 5. The throughput and delay analyzed result is shown in Figures 6 and 7.

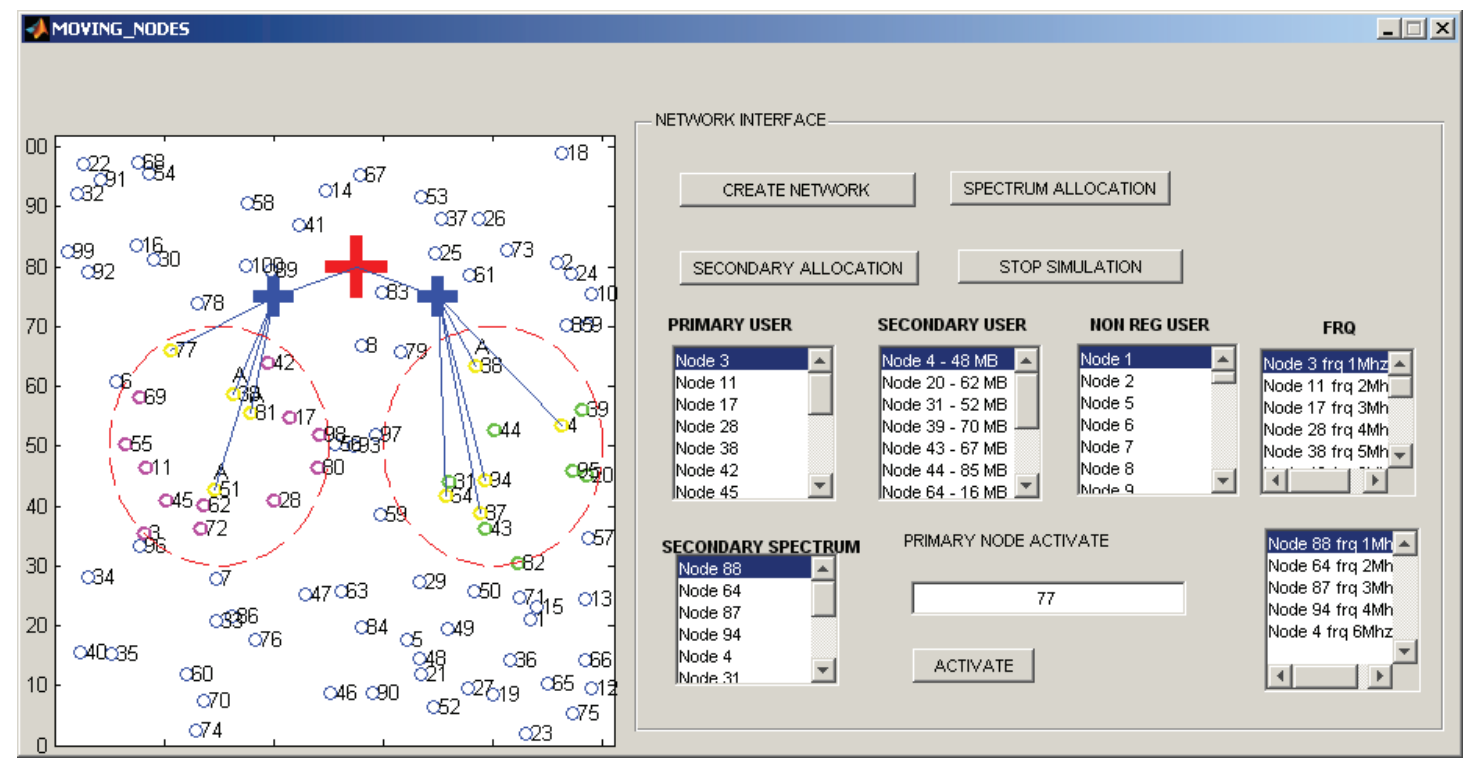

Figure 3. Cognitive Radio implementation in MATLAB.

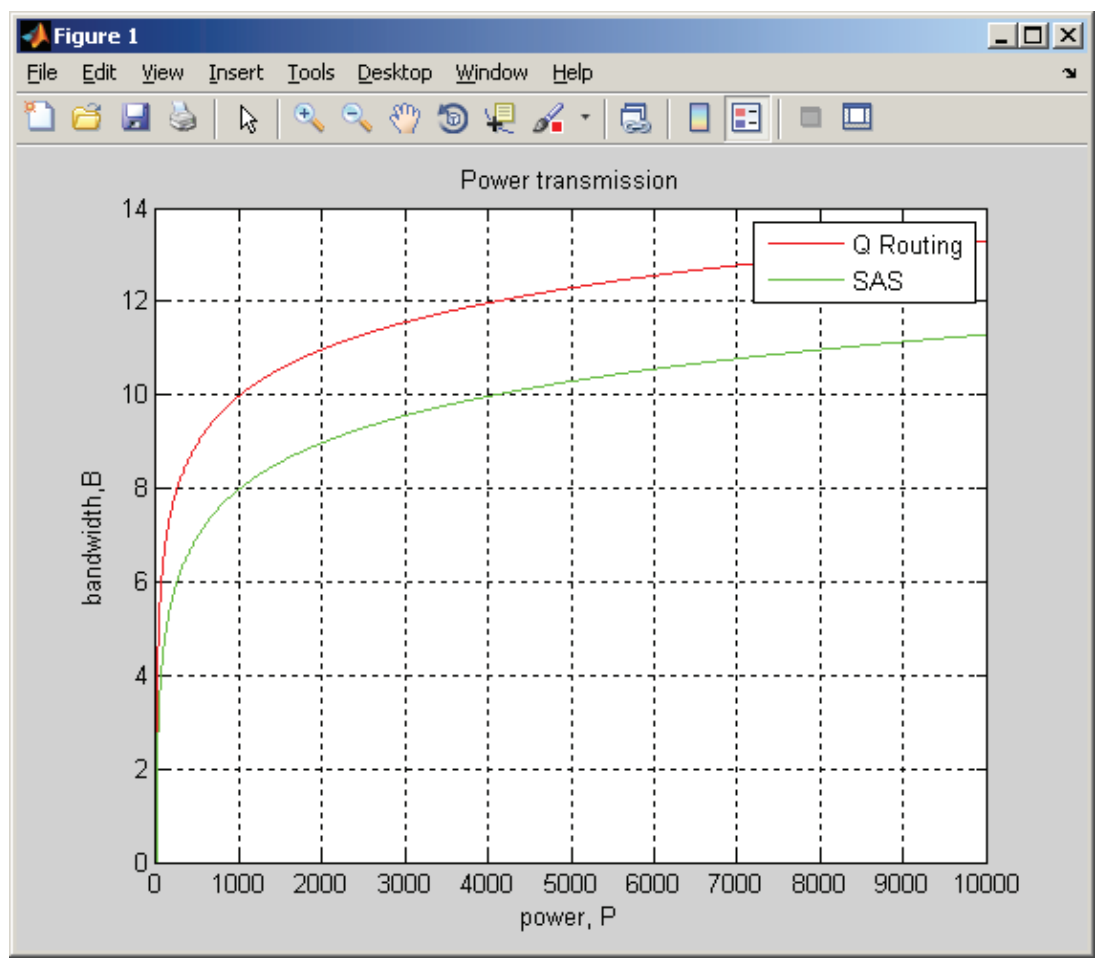

Figure 4. Transmission power. 


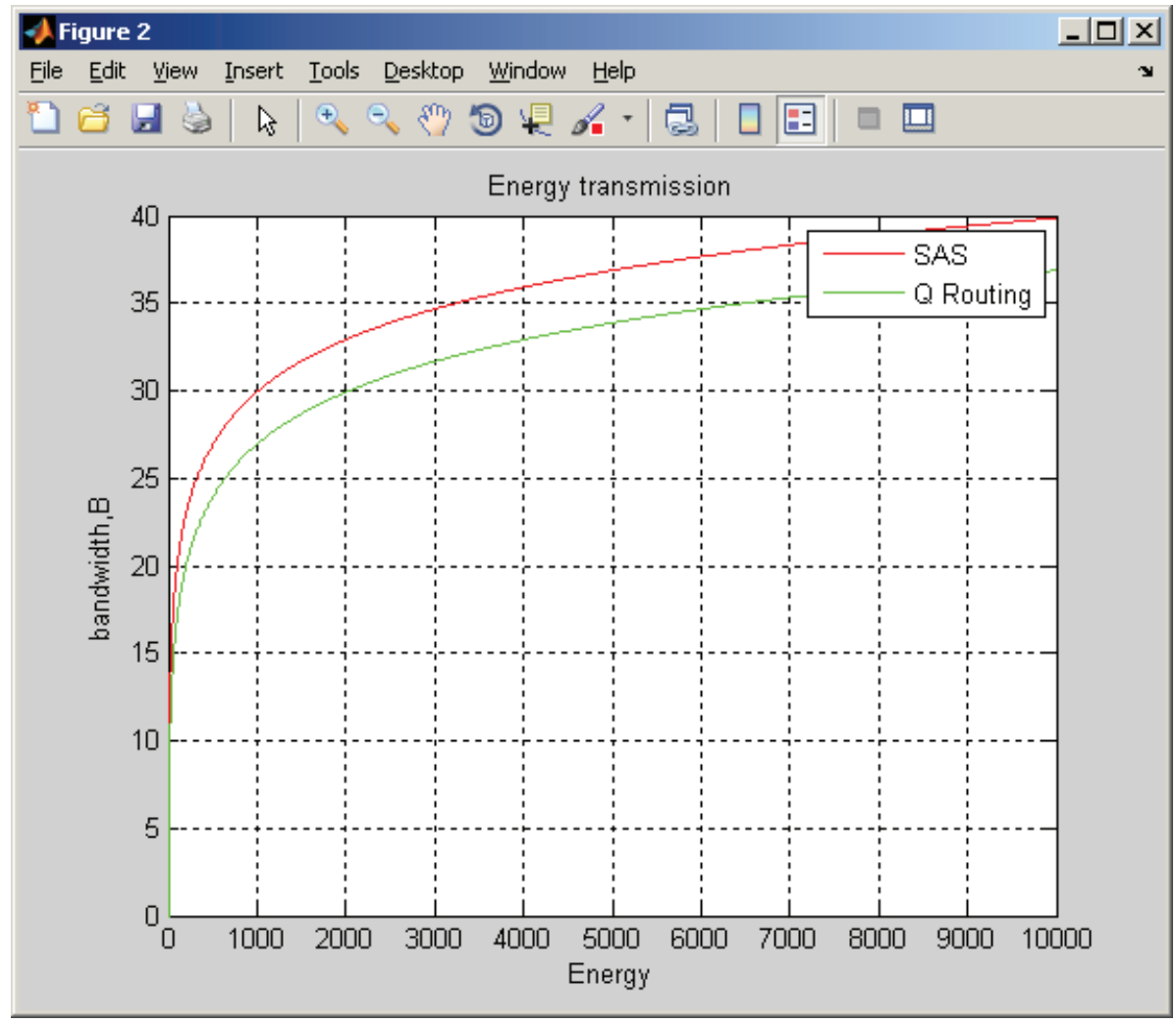

Figure 5. Energy efficiency analysis.

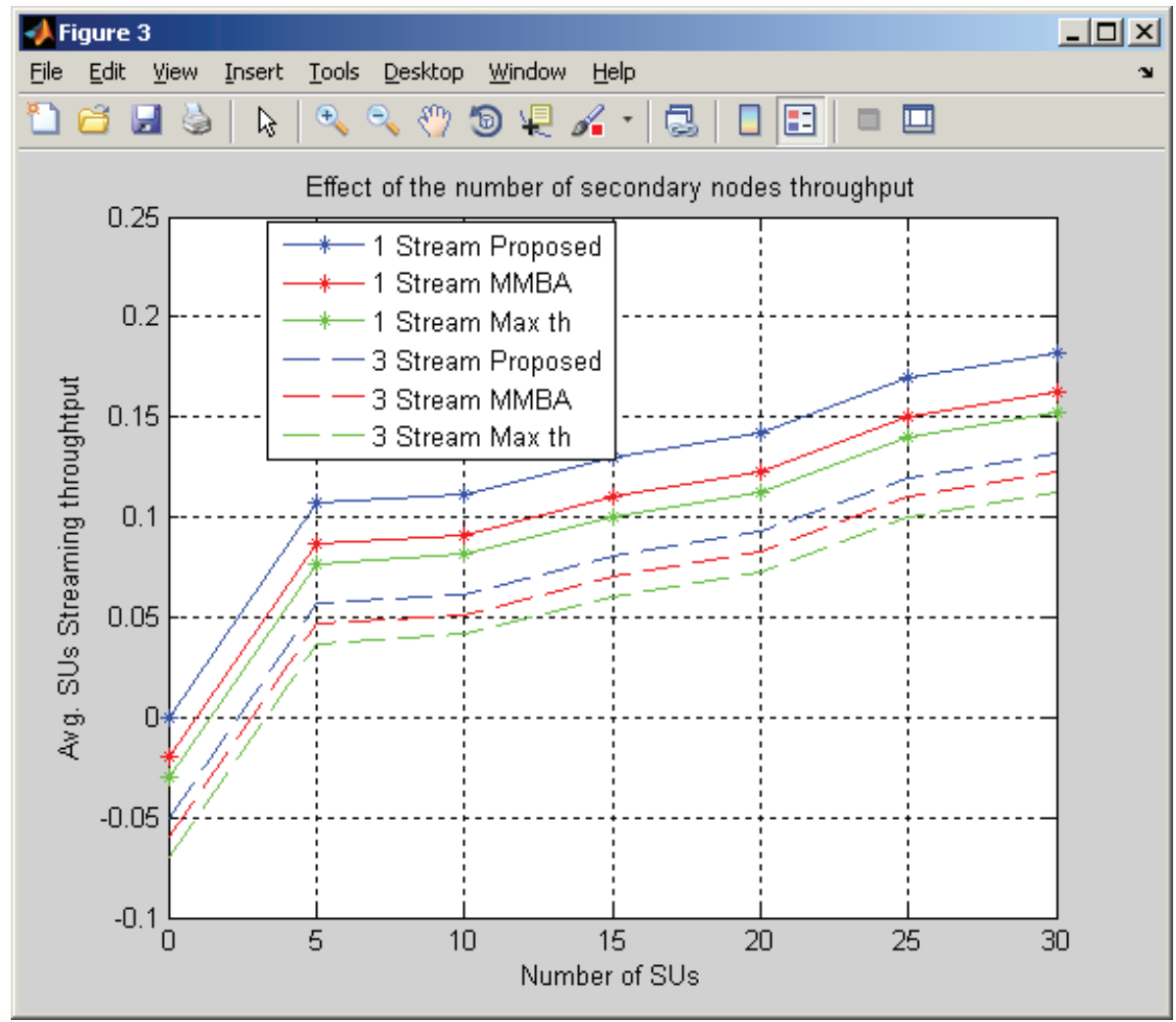

Figure 6. Throughput and Delay analysis 1. 


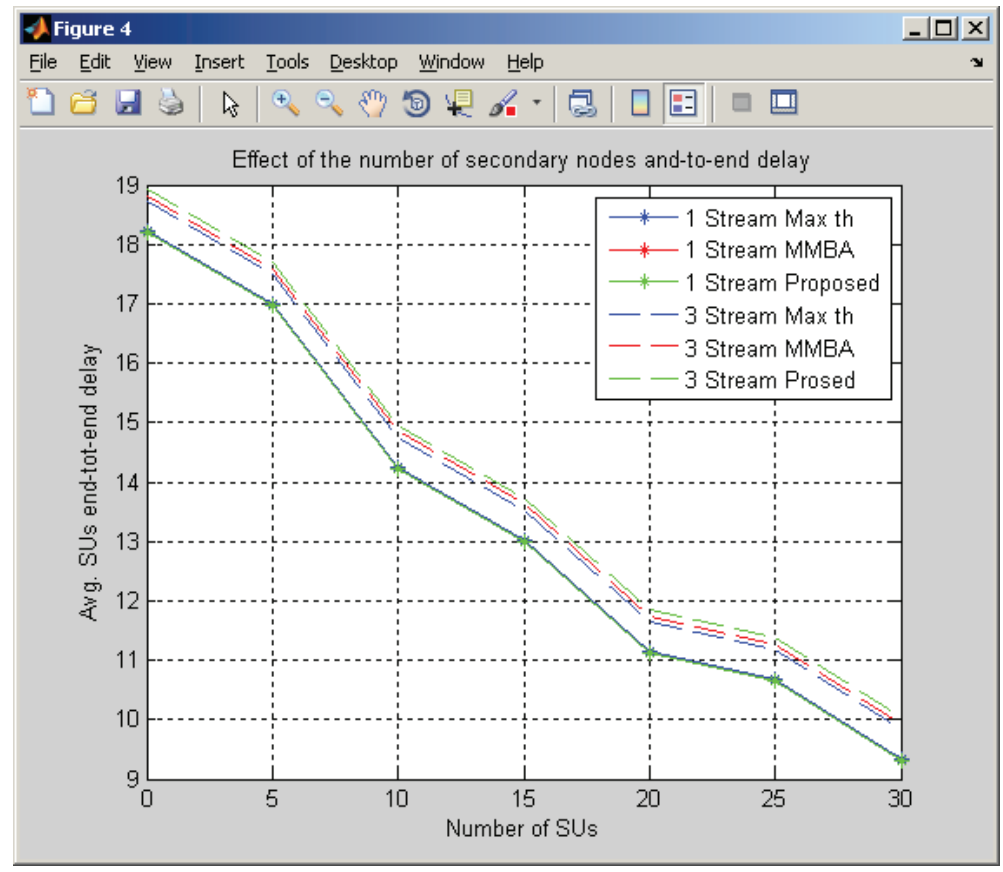

Figure 7. Throughput and Delay analysis 2.

\section{Conclusion}

In this paper, we tend to project SACRP with Stackelberg gamming theory. SACRP is given into 2 categories of cooperative routing protocols: Category A for reduced power consumption and output maximization category $\mathrm{B}$ for reducing the end-to-end delay. We have got conjointly conducted a gamming between the winning channels of the secondary user. This conjointly avoids the interference and reduces the time consumption. This enables the United States of America a path for locating the acceptable node with high strength and energy. We have a tendency to extend our approach to develop a further economical cooperative access technique of terribly very huge psychological feature radio network.

\section{References}

1. Elhachmi J, Guennoun Z. An artificial intelligence approach for cognitive spectrum management. International Journal of Computer Technology \& Applications. 2014; 5(3): 1219-25.

2. Amraoui A, Benmammar B, Krief F, Bendimerad FT. Intelligent wireless communication system using cognitive radio. International Journal of Distributed and Parallel Systems. 2012; 3(2):91-104. Crossref.
3. Gonzales CRA, Reed JH. Power fingerprinting in SDR \& CR integrity assessment. Proceeding MILCOM'09 Proceedings of the 28th IEEE conference on Military communications; 2009. p. 1089-95.

4. Deka R, Chakraborty S, Sekhar Roy J. Optimization of spectrum sensing in cognitive radio using genetic algorithm. Ser: Elec. Energ. 2012; 25(3):235-43.

5. Jegede OD, Ferens K, Kinsner W. A chaotic genetic algorithm for radio spectrum allocation. Proceedings of the International Conference on Genetic and Evolutionary Methods, Las Vegas, NV USA; 2013. p. 118-25.

6. Kaur M, Uddin M. Optimization of QoS parameters in cognitive radio using adaptive genetic algorithm. International Journal of Next-Generation Networks. 2012; 4(2):1-15. Crossref.

7. Siddique T, Azam A. Spectrum optimization in cognitive radio networks using genetic algorithms. Blenkinge Institute of Technology, Sweden; 2010.

8. Ye F, Yang R, Li Y. Genetic spectrum assignment model with constraints in cognitive radio networks. International Journal of Computer Computer Network and Information Security. 2011; 4:39-45. Crossref.

9. Varun S, Rimal S. Dynamic Resource Allocation Algorithms for Cognitive Radio Systems. Department of Electronics and Communication Engineering. 2011, pp. 1-52.

10. Saha A, Roy JS. Dynamic spectrum allocation in cognitive radio using particle swarm optimization. International Journal of Emerging Technology and Advanced Engineering; 2014. p. 54-60. 